\title{
A New Flexible Three-Parameter Model: Properties, Clayton Copula, and Modeling Real Data
}

\author{
Abdulhakim A. Al-babtain ${ }^{1}$, I. Elbatal ${ }^{2,3}$ and Haitham M. Yousof 4,* \\ 1 Department of Statistics and Operations Research, King Saud University, Riyadh 11362, Saudi Arabia; \\ hakim@ksu.edu.sa \\ 2 Department of Mathematics and Statistics, College of Science Imam Muhammad ibn Saud Islamic University, \\ Riyadh 11432, Saudi Arabia; iielbatal@imamu.edu.sa \\ 3 Department of Mathematical Statistics, Faculty of Graduate Studies for Statistical Research, Cairo University, \\ Benha 13513, Egypt \\ 4 Department of Statistics, Mathematics and Insurance, Benha University, Benha 13513, Egypt \\ * Correspondence: haitham.yousof@fcom.bu.edu.eg
}

Received: 5 February 2020; Accepted: 27 February 2020; Published: 9 March 2020

check for updates

\begin{abstract}
In this article, we introduced a new extension of the binomial-exponential 2 distribution. We discussed some of its structural mathematical properties. A simple type Copula-based construction is also presented to construct the bivariate- and multivariate-type distributions. We estimated the model parameters via the maximum likelihood method. Finally, we illustrated the importance of the new model by the study of two real data applications to show the flexibility and potentiality of the new model in modeling skewed and symmetric data sets.
\end{abstract}

Keywords: Marshall-Olkin; binomial exponential-2; moments; clayton copula; morgenstern family; maximum likelihood estimation

\section{Introduction and Motivation}

The monotonicity of the hazard (failure) rate function (HRF) of a life model plays an important role in modeling failure time data. Probability distributions with an increasing failure rate (IFR) have various applications in pricing and supply chain contracting studies. The IFR property is a well-known and useful concept in reliability theory, dynamic programming, and other areas of applied probability and statistics (See [1,2]). Recently, [3] introduced a new two-parameter lifetime model with IFR. The model of [3] is named the binomial-exponential2 (BE-2) model, which is constructed as the distribution of the random sum (RSum) of independent exponential random variables (IID RVs) when the sample size $(n)$ has a zero truncated binomial (ZTB) model. The BE-2 distribution can be used as an alternative to the standard Weibull (W), standard gamma (Ga), exponentiated exponential (EE), and weighted exponential (WhE) distributions. The cumulative distribution function (CDF) of BE-2 distribution is given by:

$$
\left.G_{\underline{\phi}}(x)\right|_{(\underline{\phi}=(\alpha, \beta))}=1-\left.e^{-\alpha x}\left(1+\frac{\beta \alpha x}{2-\beta}\right)\right|_{(x>0)},
$$

where $\alpha>0$ is the scale parameter, and $\beta$ is the shape parameter, where $0 \leq \beta \leq 1$. The probability density function (PDF) corresponding to (1) can be expressed as:

$$
g_{\underline{\phi}}(x)=\alpha\left[1+\frac{(\alpha x-1) \beta}{2-\beta}\right] e^{-\alpha x}=\frac{\alpha}{2-\beta} e^{-\alpha x}[2(1-\beta)+\alpha x \beta] .
$$

The PDF in (1) can be written as:

$$
g_{\underline{\phi}}(x)=\mathbf{P} \alpha e^{-\alpha x}+(1-\mathbf{P}) \alpha^{2} x e^{-\alpha x},
$$


where $\mathbf{P}=\frac{2(1-\beta)}{2-\beta}$. The BE-2 distribution is a mixture of the exponential (E) distribution (with scale parameter $\alpha$ ), and the Ga distribution (with shape parameter 2 and scale parameter $\alpha$ ), with mixing proportion $\mathbf{P}$. We notice that when $\beta=0$ we get the standard exponential distribution, and when $\beta=1$ the BE-2 distribution reduces to the gamma distribution with shape parameter 2 and scale parameter $\alpha$. The BE-2 distribution has a PDF whose shape is like those of $\mathrm{Ga}, \mathrm{W}, \mathrm{WhE}$, and $\mathrm{EE}$ distributions.

Recently, [4] proposed a general family of distributions called the Marshall-Olkin (MO-G) family of distributions. The MO-G family of distributions is also known as the proportional odds (PO) family. The CDF of the MO-G family is defined by:

$$
F_{\gamma_{\underline{\phi}}}(x)=\left.\frac{G_{\underline{\phi}}(x)}{1-\bar{\gamma} \bar{G}_{\underline{\phi}}(x)}\right|_{(x>0, \gamma>0)} .
$$

The survival function $(\mathrm{SF}) \bar{F}_{\gamma}(x)$ is given by:

$$
\bar{F}_{\gamma_{\underline{\phi}}}(x)=\frac{\gamma \bar{G}_{\underline{\phi}}(x)}{1-\bar{\gamma} \bar{G}_{\underline{\phi}}(x)},
$$

where $\bar{\gamma}=(1-\gamma)$, for $\gamma=1$, we get the baseline model, i.e.; $\bar{F}(x)=\bar{G}(x)$, where the shape parameter $\gamma$ is called the tilt parameter. The PDF corresponding to (4) can be expressed as:

$$
f_{\gamma_{\underline{\phi}}}(x)=\gamma g_{\underline{\phi}}(x)\left[1-\bar{\gamma} \bar{G}_{\underline{\phi}}(x)\right]^{-2},
$$

and the HRF is given by:

$$
h_{\gamma_{\underline{\prime}}}(x)=\frac{f_{\gamma_{\prime_{\underline{\phi}}}}(x)}{\bar{F}_{\gamma_{\underline{\phi}}}(x)}=\frac{\gamma g_{\underline{\phi}}(x)}{\bar{G}_{\underline{\phi}}(x)\left[1-\bar{\gamma} \bar{G}_{\underline{\phi}}(x)\right]} .
$$

The new PDF of the proposed lifetime model distribution can be right-skewed, symmetric, and left-skewed with many different useful shapes (see Figure 1), and this means that the new model will be suitable for modeling different real data sets, and the HRF of the new model exhibits many important HRF shapes such as the "increasing-constant", "decreasing", "increasing", "constant", and "bathtub" shapes (see Figure 2).

Practically, the proposed lifetime model is much better than many competitive versions of the exponential model, such as the odd Lindley exponential, the Marshall-Olkin exponential, moment exponential, the logarithmic Burr-Hatke exponential, the generalized Marshall-Olkin exponential, beta exponential, the Marshall-Olkin-Kumaraswamy exponential, the Kumaraswamy exponential, and the Kumaraswamy-Marshall-Olkin exponential, so the new lifetime model may be a good alternative to these models in modeling relief times and survival times data sets.

\section{Genesis of the New Model}

In this section, we introduce the three parameters of the MOBE-2 distribution. Using (1) and (2) in Equations (4)-(6), we obtain the CDF, SF, and PDF of the MOBE-2 distribution, (for $x>0$ ) with vector of parameters $\underline{\Psi}=(\alpha, \beta, \gamma)$. The $\mathrm{CDF}$ and SF can be written as:

$$
\begin{aligned}
& F_{\underline{\Psi}}(x)=\frac{1-\left(1+\frac{\beta \alpha x}{2-\beta}\right) e^{-\alpha x}}{1-\bar{\gamma}\left(1+\frac{\beta \alpha x}{2-\beta}\right) e^{-\alpha x}} \\
& \bar{F}_{\underline{\Psi}}(x)=\frac{\gamma\left(1+\frac{\beta \alpha x}{2-\beta}\right) e^{-\alpha x}}{1-\bar{\gamma}\left(1+\frac{\beta \alpha x}{2-\beta}\right) e^{-\alpha x}}
\end{aligned}
$$


respectively. The corresponding PDF can be derived as:

$$
f_{\underline{\Psi}}(x)=\frac{\gamma \alpha\left[1+\frac{(\alpha x-1) \beta}{2-\beta}\right] e^{-\alpha x}}{\left[1-\bar{\gamma}\left(1+\frac{\beta \alpha x}{2-\beta}\right) e^{-\alpha x}\right]^{2}} .
$$

Henceforth, let $X \sim \operatorname{MOBE}-2(\underline{\Psi})$, with PDF (10). For the MOBE-2 distribution, the HRF can be written as:

$$
h_{\underline{\Psi}}(x)=\frac{\gamma g(x)}{\bar{G}(x)[1-\bar{\gamma} \bar{G}(x)]}=\frac{\gamma \alpha\left[1+\frac{(\alpha x-1) \beta}{2-\beta}\right]}{\left(1+\frac{\beta \alpha x}{2-\beta}\right)\left[1-\bar{\gamma}\left(1+\frac{\beta \alpha x}{2-\beta}\right) e^{-\alpha x}\right]} .
$$

The MOBE-2 distribution is a very flexible model that approaches different distributions when its parameters are changed. For $=0$, the MOBE-2 distribution reduces to the Marshall-Olkin extended exponential (MOEE) distribution. For $=1$, we get MOEGa distribution with shape parameter 2 and scale parameter $\alpha$. For $=1$, we get BE-2 distribution (see Bakouch et al. (2014)). For $\beta=0$ and $=1$, we get the exponential (E) distribution. For $\beta=\gamma=1$, the MOBE-2 distribution reduces to the $\mathrm{Ga}$ model with shape parameter 2 and scale parameter $\alpha$. A useful representation for the new PDF is given in Appendix A. Figure 1 below gives some plots of the new PDF based on some selected parameters values. Based on Figure 1, we note that the new MOBE-2 distribution PDF can be right-skewed and left-skewed with many different useful shapes.
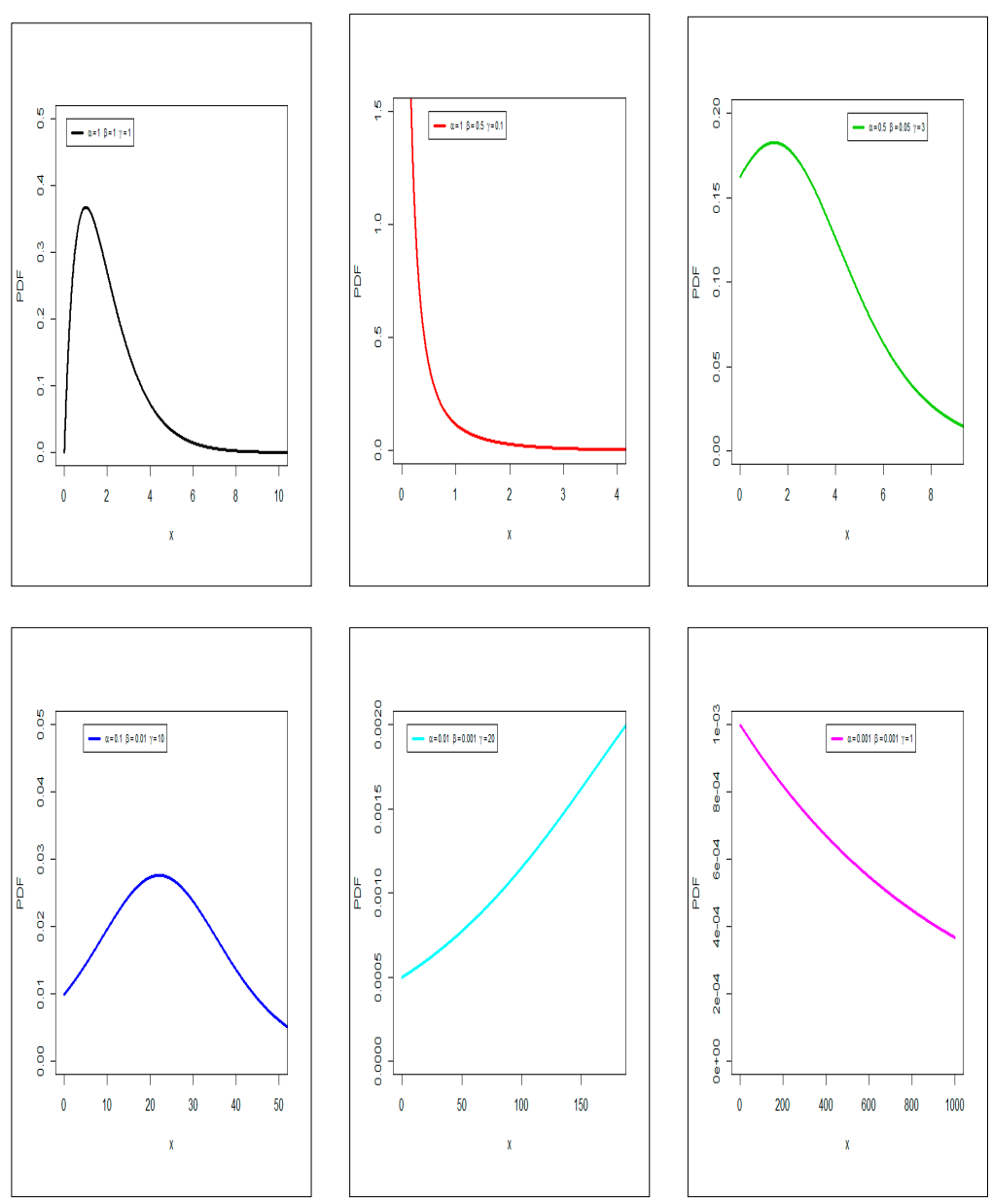

Figure 1. Plots of the new PDF based on some selected parameters values. 
Figure 2 below gives some plots of the new HRF based on some selected parameters values. From Figure 2 we note that the HRF of the new model exhibits many important HRF shapes, such as the increasing-constant $(\alpha=1, \beta=1, \gamma=1)$, decreasing $(\alpha=1, \beta=0.5, \gamma=0.1)$, increasing $(\alpha=0.005, \beta=1, \gamma=1)$, constant $(\alpha=0.001, \beta=0.001, \gamma=1)$, and bathtub $(\alpha=0.05, \beta=0.05, \gamma=0.65)$ shapes.
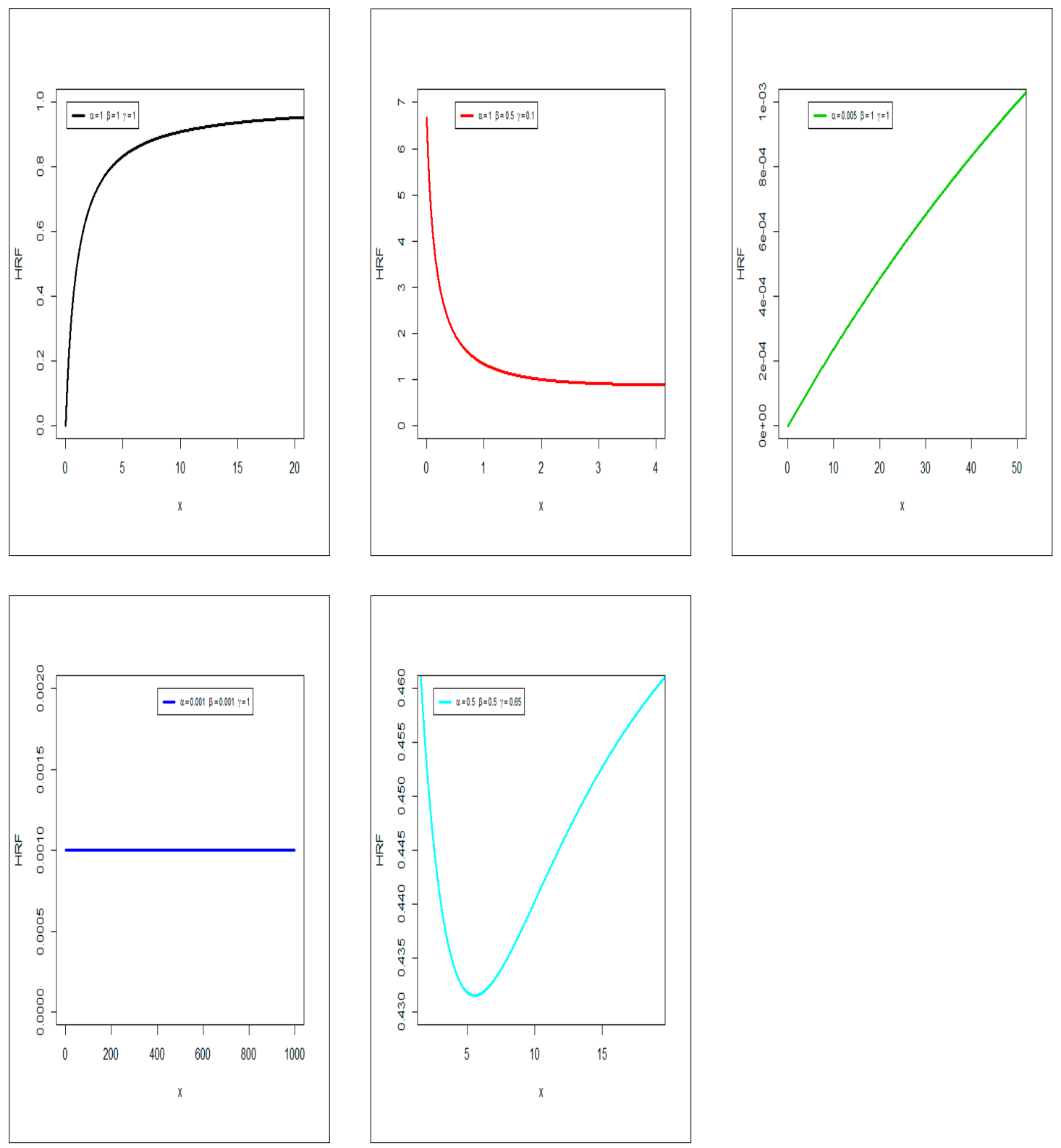

Figure 2. Plots of the new PDF based on some selected parameters values.

The solution of the following relationship is used to find the quantile function (QF) of the MOBE-2 distribution, as follows:

$$
\left(1+\frac{\beta \alpha x_{q}}{2-\beta}\right) e^{-\alpha x_{q}}-\frac{1-q}{1-\bar{\gamma} q}=0
$$

Since the uniform RVs are easily generated numerically in most statistical packages, the above scheme in (12) is very useful to generate MOBE-2 RVs and therefore can be easily implemented. It facilitates ready quantile-based statistical modeling. In particular, the median of $X$ is $Q\left(\frac{1}{2}\right)$, given by setting $q=\frac{1}{2}$ in (12). Also using (12), we can determine the Bowley's skewness and the Moors' 
kurtosis. The Bowley's skewness is based on quartiles. Figure 3 indicates that both measures depend very much on the shape parameters $\beta$.

Skewness

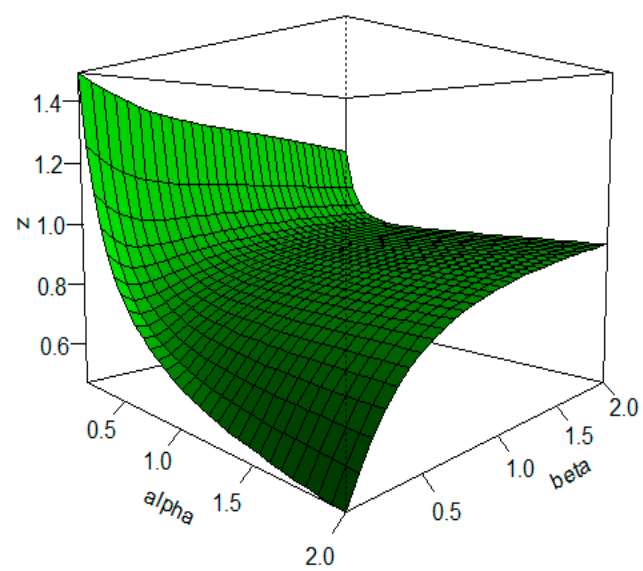

Kurtosis

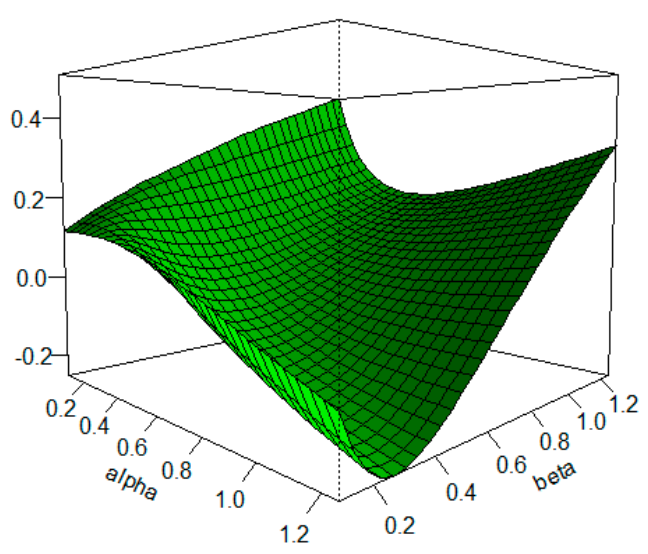

Figure 3. 3-D plot for skewness and kurtosis of the new model when $\gamma=2.25$.

\section{Properties}

\subsection{Moments}

Theorem 1. If $X \sim M O B E-2(\underline{\Psi})$, the $r^{(t h)}$ moment of $X$ is given by:

$$
\mu_{r}^{\prime}(x)=\sum_{\kappa=0}^{\infty} \sum_{\tau=0}^{\kappa} C_{\tau, \kappa} \Gamma(r+\tau),
$$

where:

$$
C_{\tau, \kappa}=\frac{w_{\tau, \kappa}}{[\alpha(1+\kappa)]^{r+\tau+2}}[2 \alpha(1-\beta)(1+\kappa)+\beta \alpha(1+\tau+r)] .
$$

Proof of Theorem 1. Let $X$ be a RV following the MOBE-2 distribution. The $r^{(t h)}$ ordinary moment can be obtained using the well-known formula:

$$
\mu_{r}^{\prime}(x)=\int_{0}^{\infty} x^{r} f_{\Psi}(x) d x .
$$

Then:

$$
\mu_{r}^{\prime}(x)=\gamma \alpha \sum_{\kappa=0}^{\infty} \sum_{\tau=0}^{\kappa} w_{\tau, \kappa} \int_{0}^{\infty} x^{r+\tau}[2(1-\beta)+\beta \alpha x] e^{-\alpha(1+\kappa) x} d x,
$$

setting $=\alpha(1+\kappa) x$, and after some algebra, the $r^{(t h)}$ ordinary moment is given by:

$$
\mu_{r}^{\prime}(x)=\sum_{\kappa=0}^{\infty} \sum_{\tau=0}^{\kappa} C_{\tau, \kappa} \Gamma(r+\tau)
$$

which complete the proof, where $\Gamma(n)=\int_{0}^{\infty} x^{n-1} e^{-x} d x$ refers to the gamma function. 
3.2. Moment Generating Function (MGF)

Theorem 2. If X has the MOBE-2 ( $\underline{\Psi})$, then the MGF of X is given as follows:

$$
M_{X}(t)=\sum_{\kappa=0}^{\infty} \sum_{\tau=0}^{\kappa} C_{\tau, \kappa}^{*} \Gamma(\tau+1),
$$

where:

$$
C_{\tau, \kappa}^{*}=\frac{w_{\tau, \kappa}}{[\alpha(1+\kappa)-t]^{\tau+2}}[2 \alpha(1-\beta)[\alpha(1+\kappa)-t]+\beta \alpha(\tau+1)]
$$

Proof of Theorem 2. The MGF can be derived from:

$$
M_{X}(t)=E\left(e^{t X}\right)=\int_{0}^{\infty} e^{t x} f(x) d x
$$

then we have:

$$
M_{X}(t)=\sum_{\kappa=0}^{\infty} \sum_{\tau=0}^{\kappa} w_{\tau, \kappa} \int_{0}^{\infty} x^{\tau}[2(1-\beta)+\beta \alpha x] e^{-[\alpha(1+\kappa-t] x} d x,
$$

which can be written as:

$$
M_{X}(t)=\sum_{\kappa=0}^{\infty} \sum_{\tau=0}^{\kappa} C_{\tau, \kappa}^{*} \Gamma(\tau+1),
$$

which completes the proof.

\subsection{Conditional Moments}

For any lifetime model, the $s^{(t h)}$ lower $\left(\delta_{s}(t)\right)$ and upper $\left(\pi_{s}(t)\right)$ IM of $X$ is defined by:

$$
\delta_{S}(t)=E\left(\left.X^{s}\right|_{(X<t)}\right)=\int_{0}^{t} x^{s} f_{\underline{\Psi}}(x) d x
$$

and

$$
\pi_{S}(t)=E\left(\left.X^{s}\right|_{(X>t)}\right)=\int_{t}^{\infty} x^{s} f_{\underline{\Psi}}(x) d x
$$

respectively, for any real $s>0$. The $s^{(t h)}$ lower incomplete moment of MOBE-2 distribution is:

$$
\begin{aligned}
\delta_{\mathcal{S}}(t) & =\int_{0}^{t} x^{s} f(x) d x=\sum_{\kappa=0}^{\infty} \sum_{\tau=0}^{\kappa} w_{\tau, \kappa} \int_{0}^{t} x^{s+\tau}[2(1-\beta)+\beta \alpha x] e^{-\alpha(1+\kappa) x} d x \\
& =\sum_{\kappa=0}^{\infty} \sum_{\tau=0}^{\kappa}(\{2 \alpha(1-\beta)(1+\kappa)[\Gamma(s+\tau+1, \alpha(1+\kappa) t]\}+\beta \alpha[\Gamma(s+\tau+2, \alpha(1+\kappa) t]),
\end{aligned}
$$

where:

$$
\Upsilon_{\tau, \kappa}=\frac{w_{\tau, \kappa}}{[\alpha(1+\kappa)]^{s+\tau+2}}
$$

and:

$$
\Gamma(s, t)=\int_{0}^{t} x^{s-1} e^{-x} d x,
$$

is the lower incomplete gamma function, where:

$$
\begin{aligned}
& \left.\Gamma(s, q)\right|_{(s \neq 0,-1,-2, \ldots)}=\int_{0}^{q} t^{s-1} \exp (-t) d t \\
& =\frac{q^{s}}{s}\left\{1 F_{1}[s ; s q+1 ;-q]\right\} \\
& =\sum_{\kappa=0}^{\infty} \frac{(-1)^{\kappa}}{\kappa !(s+\kappa)} q^{s+\kappa},
\end{aligned}
$$


the function $1 F_{1}[\cdot, \cdot, \cdot]$ is a called the confluent hypergeometric function. The first incomplete moment of $X$, denoted by, $\delta_{1}(t)$, is computed using Equation (24) by setting $s=1$ as:

$$
\begin{aligned}
\delta_{1}(t) & =\sum_{\kappa=0}^{\infty} \sum_{\tau=0}^{\kappa} V_{\tau, \kappa}(\{2 \alpha(1-\beta)(1+\kappa)[\Gamma(\tau+2, \alpha(1+\kappa) t]\} \\
& +\beta \alpha[\Gamma(\tau+3, \alpha(1+\kappa) t])
\end{aligned}
$$

where:

$$
V_{\tau, \kappa}=\gamma \alpha \frac{(1+\kappa) \bar{\gamma}^{\kappa}(\beta \alpha)^{\tau}}{(2-\beta)^{\tau+1}[\alpha(1+\kappa)]^{\tau+3}} .
$$

Similarly, the $s^{(t h)}$ upper incomplete moment of MOBE-2 distribution is:

$$
\begin{aligned}
\pi_{s}(t) & =\int_{t}^{\infty} x^{s} f(x) d x \\
& =\sum_{\kappa=0}^{\infty} \sum_{\tau=0}^{\kappa} w_{\tau, \kappa} \int_{t}^{\infty} x^{s+\tau}[2(1-\beta)+\beta \alpha x] e^{-\alpha(1+\kappa) x} d x \\
& =\sum_{\kappa=0}^{\infty} \sum_{\tau=0}^{\kappa} \Upsilon_{\tau, \kappa}(\{2 \alpha(1-\beta)(1+\kappa)[\Gamma(s+\tau+1, \alpha(1+\kappa) t]\} \\
& +\beta \alpha[\Gamma(s+\tau+2, \alpha(1+\kappa) t]),
\end{aligned}
$$

where $\Upsilon_{\tau, \kappa}=\frac{w_{\tau, \kappa}}{[\alpha(1+\kappa)]^{s+\tau+2}}$, and $\Gamma(s, t)=\int_{t}^{\infty} x^{s-1} e^{-x} d x$, is the upper incomplete gamma function. The MRL is given by $m_{X}(t)=E\left(\left.X\right|_{(X>t)}\right)=\frac{\pi_{1}(t)}{\bar{F}(t)}-t$, where $\pi_{1}(t)$ is the first incomplete moment of $X$ and by setting $s=1$ in Equation (37), we get:

$$
m_{X}(t)=\frac{1}{\bar{F}(t)} \sum_{\kappa=0}^{\infty} \sum_{\tau=0}^{\kappa} K_{\tau, \kappa}(\{2 \alpha(1-\beta)(1+\kappa) \Gamma(\tau+21, \alpha(1+\kappa) t\}+\beta \alpha \Gamma(\tau+3, \alpha(1+\kappa)-t),
$$

where $K_{\tau, \kappa}=\frac{w_{\tau, \kappa}}{[\alpha(1+\kappa)]^{\tau+3}}$. The mean inactivity time (MIT) of $X$ is defined (for $\left.t>0\right)$ by:

$$
M_{X}(t)=E(X \mid X<t)=t-\frac{\delta_{1}(t)}{F(t)} .
$$

Then, we have:

$$
M_{X}(t)=t-\frac{1}{F(t)} \sum_{\kappa=0}^{\infty} \sum_{\tau=0}^{\kappa} K_{\tau, \kappa}\left\{\begin{array}{c}
2 \alpha(1-\beta)(1+\kappa)[\Gamma(\tau+2, \alpha(1+\kappa) t] \\
+\beta \alpha[\Gamma(\tau+3, \alpha(1+\kappa) t]
\end{array}\right\}
$$

\subsection{Residual Life and Reversed Failure Rate Function}

The $n^{\text {th }}$ order moment of the residual life is given by the general formula (see [5]):

$$
\mu_{n}(t)=E\left(\left.(X-t)^{n}\right|_{(X>t)}\right)=\left.\frac{1}{\bar{F}(t)} \int_{t}^{\infty}(x-t)^{n} f_{\underline{\Psi}}(x) d x\right|_{(r \geq 1)} .
$$

Applying the binomial expansion of $(x-t)^{n}$ and substituting $f_{\underline{\Psi}}(x)$ given by (10) into the above formula gives:

$$
\begin{aligned}
\mu_{n}(t) & =\frac{1}{\bar{F}(t)} \sum_{h=0}^{n}(-t)^{n-h}\left(\begin{array}{c}
n \\
h
\end{array}\right) \int_{t}^{\infty} x^{n} f_{\underline{\Psi}}(x) d x \\
& =\frac{1}{\bar{F}(t)} \sum_{\kappa=0}^{\infty} \sum_{\tau=0}^{\kappa} \sum_{h=0}^{n} \zeta_{\tau, \kappa}\{2 \alpha(1-\beta)(1+\kappa)[\Gamma(1+n+\tau, \alpha(1+\kappa) t] \\
& +\beta \alpha[\Gamma(2+n+\tau, \alpha(1+\kappa) t]\},
\end{aligned}
$$


where $\zeta_{\tau, \kappa}=\frac{(-t)^{n-h}}{[\alpha(1+\kappa)]^{2+n+\tau}} w_{\tau, \kappa}\left(\begin{array}{l}n \\ h\end{array}\right)$. The MRL of the MOBE-2 distribution is obtained by setting $n=1$ in $\mu_{n}(t)$. The variance of the residual life of the MOBE- 2 distribution can be obtained easily by using $\mu_{2}(t)$ and $\mu(t)$. The $r$-th moment of the reversed residual life (MRRL) can be obtained by the well-known formula:

$$
m_{n}(t)=E\left(\left.(t-X)^{n}\right|_{(X \leq t)}\right)=\left.\frac{1}{F(t)} \int_{0}^{t}(t-x)^{n} f_{\underline{\Psi}}(x) d x\right|_{(n \geq 1)} .
$$

Applying the binomial expansion of $(t-x)^{n}$ and substituting $f_{\underline{\Psi}}(x)$, given before, into the above formula gives:

$$
\begin{aligned}
m_{n}(t) & =\frac{1}{F(t)} \sum_{h=0}^{n}(-t)^{n-h}\left(\begin{array}{l}
n \\
h
\end{array}\right) \int_{0}^{t} x^{n} f_{\Psi}(x) d x \\
& =\frac{1}{F(t)} \sum_{\kappa=0}^{\infty} \sum_{\tau=0}^{\kappa} \sum_{h=0}^{n} \zeta_{\tau, \kappa}\{2 \alpha(1-\beta)(1+\kappa)[\Gamma(1+n+\tau, \alpha(1+\kappa) t] \\
& +\beta \alpha[\Gamma(2+n+\tau, \alpha(1+\kappa) t]\} .
\end{aligned}
$$

The mean waiting time (MWT) of the MOBE-2 distribution can be obtained by setting $n=1$ in $m_{n}(t)$. Using $m(t)$ and $m_{2}(t)$, one can obtain the variance and the coefficient of variation of the reversed residual life of the MOBE-2 distribution (for more details see [2]).

\section{Simple Type Copula-Based Construction}

\subsection{The Bivariate MOBE-2 Using the Morgenstern Family}

First, we start with CDF for the Morgenstern family of two RVs $\left(X_{1}, X_{2}\right)$, which has the following form:

$$
\left.F_{\lambda}\left(x_{1}, x_{2}\right)\right|_{(|\lambda| \leq 1)}=F_{1}\left(x_{1}\right) F_{2}\left(x_{2}\right)\left\{1+\lambda\left[1-F_{1}\left(x_{1}\right)\right]\left[1-F_{2}\left(x_{2}\right)\right]\right\},
$$

setting:

$$
F_{\gamma_{1}, \alpha_{1}, \beta_{1}}\left(x_{1}\right)=\frac{1-\left(1+\frac{\alpha_{1} \beta_{1} x_{1}}{-\beta_{1}+2}\right) e^{-\alpha_{1} x_{1}}}{1-\bar{\gamma}_{1}\left(1+\frac{\alpha_{1} \beta_{1} x_{1}}{-\beta_{1}+2}\right) e^{-\alpha_{1} x_{1}}}
$$

and:

$$
F_{\gamma_{2}, \alpha_{2}, \beta_{2}}\left(x_{2}\right)=\frac{1-\left(1+\frac{\alpha_{2} \beta_{2} x_{2}}{-\beta_{2}+2}\right) e^{-\alpha_{2} x_{2}}}{1-\bar{\gamma}_{2}\left(1+\frac{\alpha_{2} \beta_{2} x_{2}}{-\beta_{2}+2}\right) e^{-\alpha_{2} x_{2}}},
$$

then we have a seven-dimension parameter model.

\subsection{Via Clayton Copula}

\subsubsection{The Bivariate MOBE-2 Model}

The bivariate extension via Clayton copula can be considered as a weighted version of the Clayton Copula, which is of the form

$$
C(u, v)=\left[u^{-\left(\delta_{1}+\delta_{2}\right)}+v^{-\left(\delta_{1}+\delta_{2}\right)}-1\right]^{-\frac{1}{\delta_{1}+\delta_{2}}} .
$$

This is indeed a valid copula. Next, let us assume that $X \sim \operatorname{MOBE}-2\left(\gamma_{1}, \alpha_{1}, \beta_{1}\right)$ and $Y \sim \operatorname{MOBE}-2$ $\left(\gamma_{2}, \alpha_{2}, \beta_{2}\right)$. Then, setting:

$$
u=u(x)=\frac{1-\left(1+\frac{\alpha_{1} \beta_{1} x}{-\beta_{1}+2}\right) e^{-\alpha_{1} x}}{1-\bar{\gamma}_{1}\left(1+\frac{\alpha_{1} \beta_{1} x}{-\beta_{1}+2}\right) e^{-\alpha_{1} x}},
$$


and:

$$
v=v(y)=\frac{1-\left(1+\frac{\alpha_{2} \beta_{2} y}{-\beta_{2}+2}\right) e^{-\alpha_{2} y}}{1-\bar{\gamma}_{2}\left(1+\frac{\alpha_{2} \beta_{2} y}{-\beta_{2}+2}\right) e^{-\alpha_{2} y}}
$$

the associated CDF bivariate MOBE-2 type distribution will be:

$$
H(x, y)==\left\{\begin{array}{c}
{\left[\frac{1-\left(1+\frac{\alpha_{1} \beta_{1} x}{-\beta_{1}+2}\right) e^{-\alpha_{1} x}}{1-\bar{\gamma}_{1}\left(1+\frac{\alpha_{1} \beta_{1} x}{-\beta_{1}+2}\right) e^{-\alpha_{1} x}}\right]^{-\left(\delta_{1}+\delta_{2}\right)}} \\
+\left[\frac{1-\left(1+\frac{\alpha_{2} \beta_{2} y}{-\beta_{2}+2}\right) e^{-\alpha_{2} y}}{1-\bar{\gamma}_{2}\left(1+\frac{\alpha_{2} \beta_{2} y}{-\beta_{2}+2}\right) e^{-\alpha_{2} y}}\right]^{-\left(\delta_{1}+\delta_{2}\right)} \\
-1
\end{array}\right\}^{-\frac{1}{\delta_{1}+\delta_{2}}} .
$$

Note: depending on the specific baseline $\mathrm{CDF}$, one may construct various bivariate MOBE-2 type models in which $\left(\delta_{1}+\delta_{2}\right) \geq 0$.

\subsubsection{The Multivariate Extension}

The $d$-dimensional version from the above will be:

$$
H\left(x_{1}, x_{2}, \cdots, x_{d}\right)=\left\{\sum_{i=1}^{d}\left[\frac{1-\left(1+\frac{\alpha_{i} \beta_{i} x_{i}}{-\beta_{1}+2}\right) e^{-\alpha_{i} x_{i}}}{1-\bar{\gamma}_{i}\left(1+\frac{\alpha_{i} \beta_{i} x_{i}}{-\beta_{i}+2}\right) e^{-\alpha_{i} x_{i}}}\right]^{-\left(\delta_{1}+\delta_{2}\right)}+1-d\right\}^{-1 /\left(\delta_{1}+\delta_{2}\right)} .
$$

Further future works could be allocated for studying the bivariate and the multivariate extensions of the MOBE-2 model.

\section{Estimation and Inference}

Let $X_{1}, X_{2}, \ldots, X_{n}$ be a random sample of size $n$ from MOBE-2 $(\underline{\Psi})$ where $\underline{\Psi}=(\alpha, \beta, \gamma)$. The log likelihood function $\left(\log L_{(\underline{\Psi})}\right)$ can be written as:

$$
\begin{aligned}
\log L_{(\underline{\Psi})} & =n \log (\alpha)+n \log (\gamma)-n \log (2-\beta)+\sum_{i=0}^{n} \log \left[2-2 \beta+\beta \alpha x_{i}\right]-\alpha \sum_{i=0}^{n} x_{i} \\
& -2 \sum_{i=0}^{n} \log \left[1-\bar{\gamma}\left(1+\frac{\beta \alpha x_{i}}{2-\beta}\right) e^{-\alpha x_{i}}\right] .
\end{aligned}
$$

The associated score function is given by $U_{n}(\underline{\Psi})=\left(\frac{\partial \log L_{(\underline{\Psi}}}{\partial \alpha}, \frac{\partial \log L_{(\underline{\Psi})}}{\partial \beta}, \frac{\partial \log L_{(\Psi)}}{\partial \gamma}\right)^{T}$. The $\log$-likelihood can be maximized either directly or by solving the nonlinear likelihood equations obtained by differentiating $\log L_{(\underline{\Psi})}$. The components of the score vector are given by:

$$
\begin{gathered}
\frac{\partial \log L_{(\underline{\Psi})}}{\partial \alpha}=\frac{n}{\alpha}+\sum_{i=0}^{n} \frac{\beta x_{i}}{2-2 \beta+\beta \alpha x_{i}}-\sum_{i=0}^{n} x_{i}+2 \bar{\gamma} \sum_{i=0}^{n} \frac{\frac{\beta x_{i} e^{-\alpha x_{i}}}{2-\beta}-x_{i} e^{-\alpha x_{i}}\left(1+\frac{\beta \alpha x_{i}}{2-\beta}\right)}{1-\bar{\gamma}\left(1+\frac{\beta \alpha x_{i}}{2-\beta}\right) e^{-\alpha x_{i}}}, \\
\frac{\partial \log L_{(\underline{\Psi})}}{\partial \beta}=\frac{n}{2-\beta}+\sum_{i=0}^{n} \frac{\alpha x_{i}-2}{2-2 \beta+\beta \alpha x_{i}}+4 \sum_{i=0}^{n} \frac{\bar{\gamma} \alpha x_{i}}{(2-\beta)^{2}\left[1-\bar{\gamma}\left(1+\frac{\beta \alpha x_{i}}{2-\beta}\right) e^{-\alpha x_{i}}\right]},
\end{gathered}
$$

and:

$$
\frac{\partial \log L_{(\underline{\Psi})}}{\partial \gamma}=\frac{n}{\gamma}-2 \sum_{i=0}^{n} \frac{\left(1+\frac{\beta \alpha x_{i}}{2-\beta}\right) e^{-\alpha x_{i}}}{1-\bar{\gamma}\left(1+\frac{\beta \alpha x_{i}}{2-\beta}\right) e^{-\alpha x_{i}}}
$$




\section{Modeling}

In this Section, two real data applications are presented for illustrating the importance and flexibility of the new model. The first data set $(1.1,1.4,1.3,1.7,1.9,1.8,1.6,2.2,1.7,2.7,4.1,1.8,1.5,1.2$, $1.4,3,1.7,2.3,1.6,2)$, called the failure time data, represents the lifetime data relating to relief times (in minutes) of patients receiving an analgesic (for more applications to this data see [6-12]).

The second data set $(0.1,0.33,0.44,0.56,0.59,0.72,0.74,0.77,0.92,0.93,0.96,1,1,1.02,1.05,1.07$, $07,1.08,1.08,1.08,1.09,1.12,1.13,1.15,1.16,1.2,1.21,1.22,1.22,1.24,1.3,1.34,1.36,1.39,1.44,1.46,1.53$, $1.59,1.6,1.63,1.63,1.68,1.71,1.72,1.76,1.83,1.95,1.96,1.97,2.02,2.13,2.15,2.16,2.22,2.3,2.31,2.4,2.45$, $2.51,2.53,2.54,2.54,2.78,2.93,3.27,3.42,3.47,3.61,4.02,4.32,4.58,5.55)$ represents the survival times (in days) of 72 guinea pigs infected with virulent tubercle bacilli, observed and reported by Bjerkedal (1960). Many other real data sets related to failure times can be found in [13-20].

Figure 3 gives the total time test (TTT) plots. Figures 4 and 5 gives the estimated PDF (EPDF), estimated survival function (ESF), P-P plots, and estimated CDF (ECDF) for the two data sets, respectively. From Figure 3, we note that the empirical HRF is increasing for the two data sets.

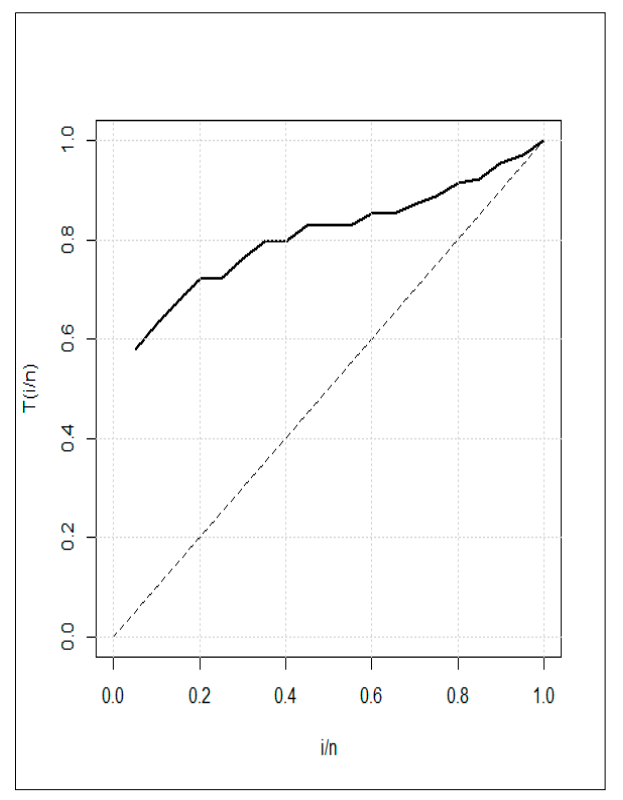

Data I

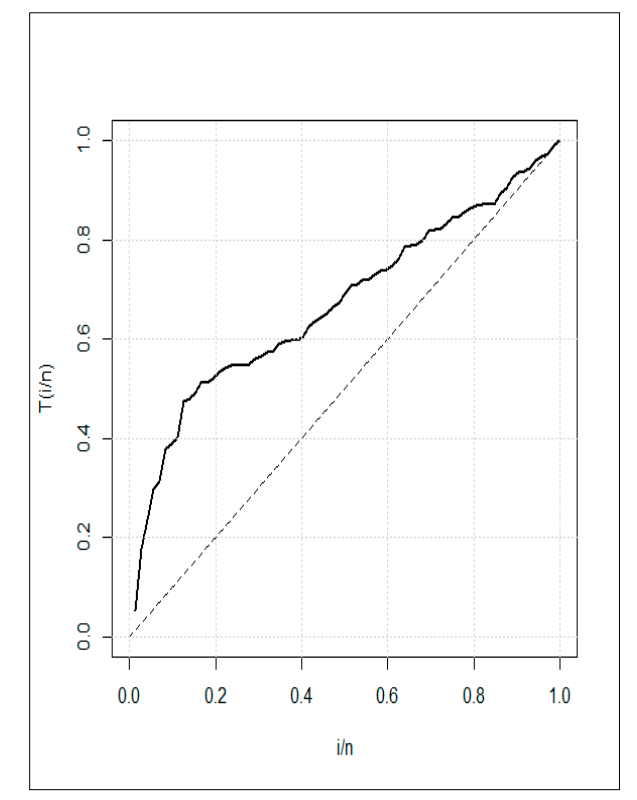

Data II

Figure 4. The total time test TTT plots.

We compared the fits of the MOBE-2 distribution with some competitive models, namely: exponential $(\mathrm{E}(\beta))$, odd Lindley exponential (OLiE), MO exponential ( $\mathrm{MOE}(\alpha, \beta))$, moment exponential $(\operatorname{MomE}(\beta))$, the logarithmic Burr-Hatke exponential $(\log \operatorname{BrHE}(\beta))$, generalized MO exponential $(\operatorname{GMOE}(\alpha, \alpha, \beta))$, beta exponential (BE $(a, b, \beta))$, MO-Kumaraswamy exponential (MOKwE $(\alpha, a, b, \beta)$ ), Kumaraswamy exponential $(\operatorname{KwE}(a, b, \beta))$, and Kumaraswamy MO exponential $(\operatorname{KwMOE}(\alpha, a, b, \beta))$. See the PDFs of the competitive moels in [21-31]. We considered the Cramér-Von Mises $\left(W^{*}\right)$, the Anderson-Darling $\left(A^{*}\right)$, and the Kolmogorov-Smirnov (KS) statistics. The $W^{*}$ and $A^{*}$ statistics are given by:

$$
W^{*}=(1+1 / 2 n)\left[[1 /(12 n)]+\sum_{\kappa=1}^{n} \omega_{\kappa}\right]
$$

and:

$$
A^{*}=a_{(n)}\left(n+n^{-1} \sum_{\kappa=1}^{n} a_{\kappa}\right)
$$


where:

$$
\omega_{\mathcal{K}}=\left[z_{i}-(2 \kappa-1) /(2 n)\right]^{2}, a_{(n)}=1+\frac{9}{4} n^{-2}+\frac{3}{4} n^{-1},
$$

and:

$$
a_{\kappa}=(2 \kappa-1) \log \left[z_{i}\left(1-z_{n-1+\kappa}\right)\right],
$$

where $z_{i}=F\left(y_{\kappa}\right)$ and $y_{\kappa}$ 's values are the ordered observations. Moreover, we considered some other goodness-of-fit measures, including the Akaike Information Criterion (AIC), Consistent Akaike Information Criterion (CAIC), Hannan-Quinn Information Criterion (HQIC), and Bayesian Information Criterion (BIC).

Table 1 gives the maximum likelihood estimations (MLE) and SE values for the relief times data. Table 2 give the AIC, BIC, CAIC, and HQIC for the relief times data. Table 3 gives $A^{*}, W^{*}, \mathrm{KS}$, and $p$-value for the relief times data. Table 4 gives the MLE and SE values for the survival times data. Table 5 gives the AIC, BIC, CAIC, and HQIC for the survival times data. Table 6 gives the $A^{*}, W^{*}, \mathrm{KS}$, and $p$-value for the survival times data.

\begin{tabular}{|c|c|}
\hline Models & Estimates \\
\hline $\mathrm{E}(\beta)$ & $\begin{array}{c}0.526 \\
(0.117)\end{array}$ \\
\hline $\mathrm{OLiE}(\beta)$ & $\begin{array}{c}0.6044 \\
(0.0535)\end{array}$ \\
\hline $\operatorname{MomE}(\beta)$ & $\begin{array}{l}0.950 \\
(0.150)\end{array}$ \\
\hline $\log \operatorname{BrHE}(\beta)$ & $\begin{array}{l}0.5263 \\
(0.118)\end{array}$ \\
\hline $\operatorname{MOE}(\alpha, \beta)$ & $\begin{array}{c}54.474,2.316 \\
(35.582),(0.374)\end{array}$ \\
\hline $\operatorname{GMOE}(\alpha, \alpha, \beta)$ & $\begin{array}{c}0.519,89.462,3.169 \\
(0.256),(66.278),(0.772)\end{array}$ \\
\hline $\mathrm{KwE}(\mathrm{a}, \mathrm{b}, \beta)$ & $\begin{array}{c}83.756,0.568,3.330 \\
(42.361),(0.326),(1.188)\end{array}$ \\
\hline $\mathrm{BE}(\mathrm{a}, \mathrm{b}, \beta)$ & $\begin{array}{c}81.633,0.542,3.514 \\
(120.41),(0.327),(1.410)\end{array}$ \\
\hline $\operatorname{MOKwE}(\alpha, a, b, \beta)$ & $\begin{array}{c}0.133,33.232,0.571,1.669 \\
(0.332),(57.837),(0.721),(1.814)\end{array}$ \\
\hline $\operatorname{KwMOE}(\alpha, a, b, \beta)$ & $\begin{array}{c}8.868,34.826,0.299,4.899 \\
(9.146),(22.312),(0.239),(3.176)\end{array}$ \\
\hline $\operatorname{BrXE}(\alpha, \beta)$ & $\begin{array}{c}1.1635,0.3207 \\
(0.33),(0.03)\end{array}$ \\
\hline $\operatorname{MOBE} 2(\gamma, \alpha, \beta)$ & $\begin{array}{c}1.83 \times 10^{3}, 6.707 \times 10^{-2}, 6.096 \times 10^{-3} \\
\left(2.206 \times 10^{3}\right),\left(4.991 \times 10^{-3}\right),\left(1.069 \times 10^{-3}\right)\end{array}$ \\
\hline
\end{tabular}

Table 1. MLE and SE values for the relief times data.

Table 2. Akaike Information Criterion (AIC), Consistent Akaike Information Criterion (CAIC), Hannan-Quinn Information Criterion (HQIC), and Bayesian Information Criterion (BIC) for the relief times data.

\begin{tabular}{cc}
\hline Models & AIC, BIC, CAIC, HQIC \\
\hline MOBE2 & $\mathbf{4 0 . 1}, \mathbf{4 0 . 2}, \mathbf{4 0 . 3}, \mathbf{3 9 . 1}$ \\
E & $67.67,68.67,67.89,67.87$ \\
OLiE & $49.1,50.1,49.3,49.3$ \\
MomE & $54.32,55.31,54.54,54.50$ \\
Log BrHE & $67.67,68.67,67.89,67.87$ \\
MOE & $43.51,45.51,44.22,43.90$ \\
GMOE & $42.75,45.74,44.25,43.34$ \\
KwE & $41.78,44.75,43.28,42.32$ \\
BE & $43.48,46.45,44.98,44.02$ \\
MOKwE & $41.58,45.54,44.25,42.30$ \\
KwMOExp & $42.8,46.84,45.55,43.60$ \\
BrXE & $48.1,50.1,8.8,48.5$ \\
\hline
\end{tabular}


Based on Tables 2, 3, 5 and 6, the proposed lifetime MOBE-2 model is much better than many competitive models, such as the E, MomE, MOE, GMOE, KwE, BE, MOKE, and KMOE models, so the new lifetime model may be a good alternative to these models in modeling relief times and survival times data sets. From Figures 5 and 6, we note that the MOBE-2 model gives an adequate fit with the two real data sets.
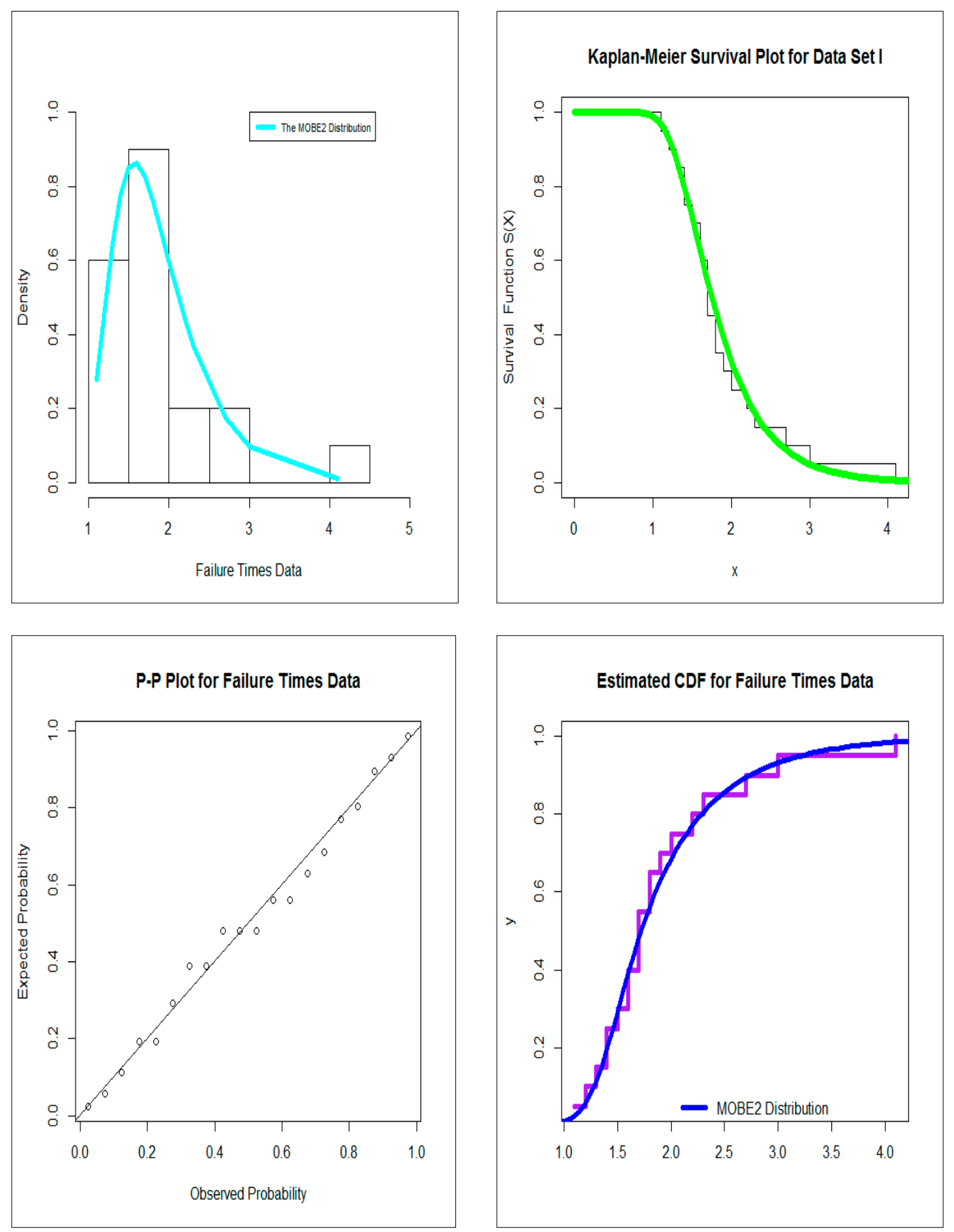

Figure 5. Estimated PDF (EPDF), estimated survival function (ESF), Probability-Probability (P-P) plot, and estimated cumulative distribution function (ECDF) for the relief times data. 
Table 3. Cramér-Von Mises $\left(W^{*}\right)$, the Anderson-Darling $\left(A^{*}\right)$, and the Kolmogorov-Smirnov (KS) statistics, and $p$-value for the relief times data.

\begin{tabular}{cc}
\hline Models & $A^{*}, \boldsymbol{W}^{*}$, KS and $p$-Value \\
\hline MOBE2 & $\mathbf{0 . 3 3}, \mathbf{0 . 0 4 6}, \mathbf{0 . 1 2}(\mathbf{0 . 9 5 )}$ \\
E & $4.60,0.96,0.44(0.004)$ \\
OLiE & $1.3,0.22,0.85\left(6.23 \times \mathrm{e}^{-13}\right)$ \\
MomE & $2.76,0.53,0.32(0.07)$ \\
Log BrHE & $0.62,0.105,0.44(0.0009)$ \\
MOE & $0.8,0.14,0.1(0.55)$ \\
GMOE & $0.51,0.08,0.15(0.78)$ \\
KwE & $0.45,0.07,0.14(0.86)$ \\
BE & $0.70,0.12,0.16(0.80)$ \\
MOKwE & $0.60,0.11,0.14(0.87)$ \\
KwOExp & $1.08,0.19,0.15(0.86)$ \\
BrXE & $1.39,0.24,0.248(0.1705)$ \\
\hline
\end{tabular}

Table 4. MLEs and SEs values for the survival times data.

\begin{tabular}{|c|c|}
\hline Models & Estimates \\
\hline $\mathrm{E}(\beta)$ & $\begin{array}{c}0.540 \\
(0.063)\end{array}$ \\
\hline $\mathrm{OLiE}(\beta)$ & $\begin{array}{l}0.38145 \\
(0.0209)\end{array}$ \\
\hline $\operatorname{MomE}(\beta)$ & $\begin{array}{c}0.925 \\
(0.077)\end{array}$ \\
\hline $\log \operatorname{BrHE}(\beta)$ & $\begin{array}{c}0.54 \\
(0.064)\end{array}$ \\
\hline $\operatorname{MOE}(\alpha, \beta)$ & $\begin{array}{c}8.778,1.379 \\
(3.555),(0.193)\end{array}$ \\
\hline $\operatorname{GMOE}(\alpha, \alpha, \beta)$ & $\begin{array}{c}0.179,47.635,4.465 \\
(0.070),(44.901),(1.327)\end{array}$ \\
\hline $\mathrm{KwE}(\mathrm{a}, \mathrm{b}, \beta)$ & $\begin{array}{c}3.304,1.100,1.037 \\
(1.106),(0.764),(0.614)\end{array}$ \\
\hline $\mathrm{BE}(\mathrm{a}, \mathrm{b}, \beta)$ & $\begin{array}{c}0.807,3.461,1.331 \\
(0.696),(1.003),(0.855)\end{array}$ \\
\hline $\operatorname{MOKwE}(\alpha, a, b, \beta)$ & $\begin{array}{c}0.008,2.716,1.986,0.099 \\
(0.002), 1.316),(0.784),(0.048)\end{array}$ \\
\hline $\operatorname{KwMOE}(\alpha, a, b, \beta)$ & $\begin{array}{c}0.373,3.478,3.306,0.299 \\
(0.136),(0.861),(0.779),(1.112)\end{array}$ \\
\hline $\operatorname{BrXE}(\alpha, \beta)$ & $\begin{array}{c}0.475,0.2055 \\
(0.06),(0.012)\end{array}$ \\
\hline $\operatorname{MOBE} 2(\gamma, \alpha, \beta)$ & $\begin{array}{c}11.0365,0.12054,0.013601 \\
(4.8066),(0.02246),(0.0077)\end{array}$ \\
\hline
\end{tabular}

Table 5. AIC, BIC, CAIC, and HQIC for the survival times data.

\begin{tabular}{cc}
\hline Models & AIC, BIC, CAIC, HQIC \\
\hline MOBE2 & $\mathbf{2 0 7 . 3 , 2 1 3 . 1 5 , 2 0 6 . 6 , 2 0 9 . 0 1}$ \\
E & $234.63,236.91,234.68,235.54$ \\
OLiE & $229.1,231.4,229.2,230$ \\
MomE & $210.40,212.68,210.45,211.30$ \\
Log BrHE & $234.63,236.9,234.7,235.5$ \\
MOE & $210.36,214.92,210.53,212.16$ \\
GMOE & $210.54,217.38,210.89,213.24$ \\
KwE & $209.42,216.24,209.77,212.12$ \\
BE & $207.38,214.22,207.73,210.08$ \\
MOKwE & $209.44,218.56,210.04,213.04$ \\
KwMOE & $207.82,216.94,208.42,211.42$ \\
BrXE & $235.3,239.9,235.5,237.1$ \\
\hline
\end{tabular}


Table 6. $A^{*}, W^{*}, \mathrm{KS}$, and $p$-value for the survival times data.

\begin{tabular}{cc}
\hline Models & $A^{*}, W^{*}, \mathbf{K S}, \boldsymbol{p}$-Value \\
\hline MOBE2 & $\mathbf{0 . 6 8}, \mathbf{0 . 0 9}, \mathbf{0 . 0 8 9 ( 0 . 6 4 )}$ \\
E & $6.53,1.25,0.27(0.06)$ \\
OLiE & $1.94,0.33,0.49\left(9.992 \times \mathrm{e}^{-16}\right)$ \\
MomE & $1.52,0.25,0.14(0.13)$ \\
Log BrHE & $0.71,0.115,0.28\left(2.382 \times \mathrm{e}^{-5}\right)$ \\
MOE & $1.18,0.17,0.1(0.43)$ \\
GMOE & $1.02,0.16,0.09(0.51)$ \\
KwE & $0.74,0.11,0.09(0.50)$ \\
BE & $0.98,0.15,0.11(0.34)$ \\
MOKwE & $0.79,0.12,0.10(0.44)$ \\
KwMOE & $0.61,0.11,0.09(0.53)$ \\
BrXE & $2.9,0.52,0.22(0.002)$ \\
\hline
\end{tabular}
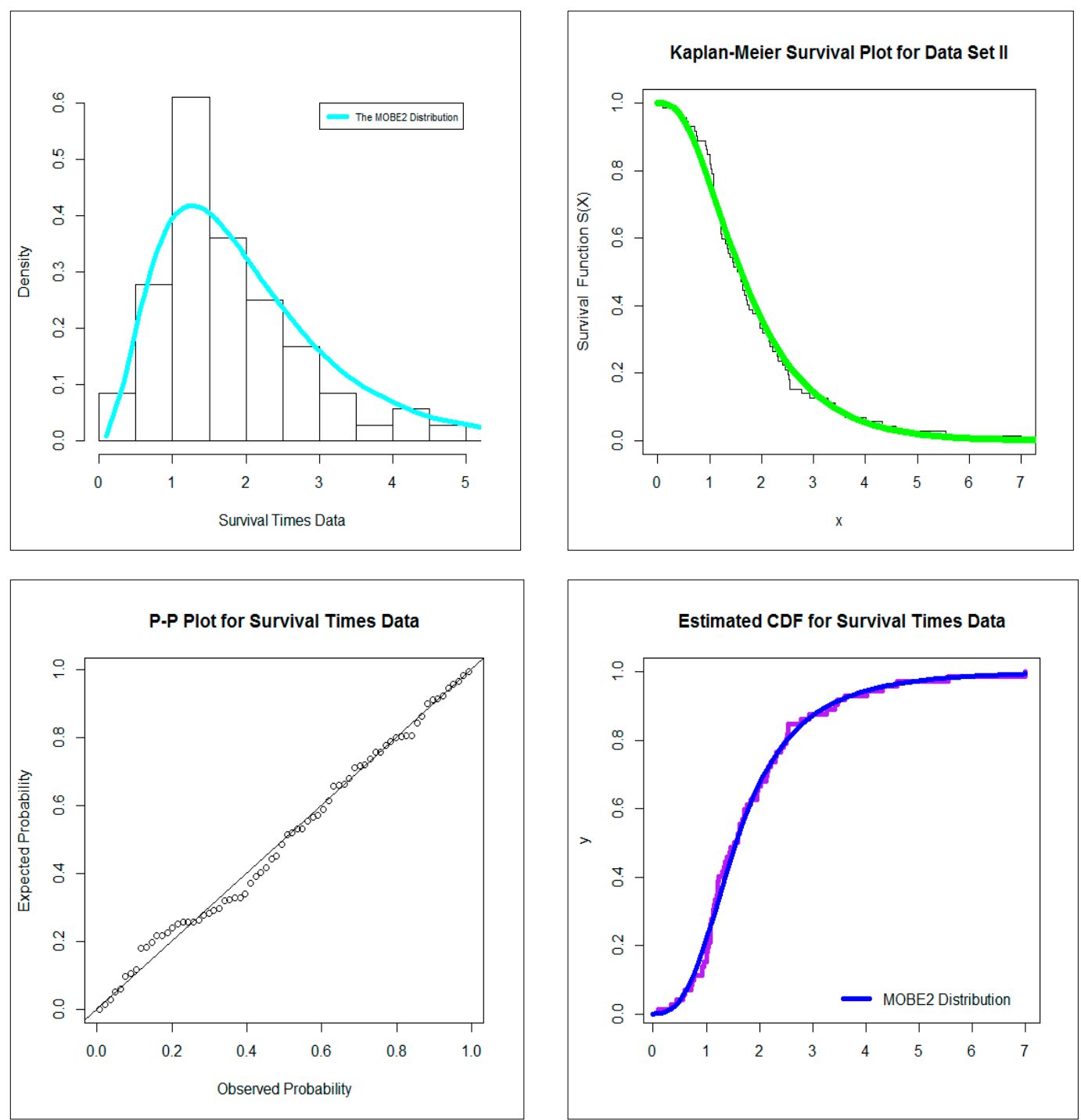

Figure 6. Estimated PDF (EPDF), estimated survival function (ESF), Probability-Probability (P-P) plot, and estimated cumulative distribution function (ECDF) for the survival times data. 


\section{Concluding Remarks}

In this paper, we introduced a new version of the BE-2 model. The new model is called MOBE-2 distribution. Some of its structural properties are also presented. A simple type Copula-based construction was also presented to construct the bivariate and the multivariate type distributions. We illustrated the importance of the new version by the study of two real data applications. The proposed lifetime model was much better than many competitive versions, such as the exponential, the odd Lindley exponential, the Marshall-Olkin exponential, moment exponential, the logarithmic Burr-Hatke exponential, the generalized Marshall-Olkin exponential, beta exponential, the Marshall-Olkin-Kumaraswamy exponential, the Kumaraswamy exponential, and the Kumaraswamy-Marshall-Olkin Exponential, so the new lifetime model may be a good alternative to these models in modeling relief times and survival times data sets.

Author Contributions: A.A.A.-b. and I.E.: writing, conceptualization, and methodology. H.M.Y.: writing-review, editing, and project administration. All authors have read and agreed to the published version of the manuscript.

Funding: This project was supported by King Saud University, Deanship of Scientific Research, and College of Sciences Research Center.

Conflicts of Interest: The authors declare no conflict of interest.

\section{Appendix A}

In this Appendix, we derive a useful expansion for the MOBE-2 density function as follows. Using the generalized binomial expression

$$
(1-z)^{-c}=\left.\sum_{\kappa=0}^{\infty} \frac{\Gamma(c+\kappa)}{\Gamma(c) \kappa !} z^{\kappa}\right|_{(|z|<1 \text { and } c>0 \text { real non-integer })}
$$

Then using (12), the PDF of the MOBE-2 can be written as

$$
f_{\underline{\Psi}}(x)=\gamma \alpha \sum_{\kappa=0}^{\infty} \bar{\gamma}^{\kappa}(1+\kappa)\left(1+\frac{\beta \alpha x}{2-\beta}\right)^{\kappa}\left[1+\frac{(\alpha x-1) \beta}{2-\beta}\right] e^{-\alpha(1+\kappa) x},
$$

applying the binomial expression for $\left(1+\frac{\beta \alpha x}{2-\beta}\right)^{\kappa}$, the new PDF becomes

$$
f_{\underline{\Psi}}(x)=\gamma \alpha \sum_{\kappa=0}^{\infty} \bar{\gamma}^{\kappa}(1+\kappa) \sum_{\tau=0}^{\kappa}\left(\frac{\beta \alpha}{2-\beta}\right)^{\tau} x^{\tau}\left[1+\frac{(\alpha x-1) \beta}{2-\beta}\right] e^{-\alpha(1+\kappa) x},
$$

after some simplification, MOBE-2 density function can be expressed as

$$
f_{\underline{\Psi}}(x)=\sum_{\kappa=0}^{\infty} \sum_{\tau=0}^{\kappa} w_{\tau, \kappa} x^{\tau}[2(1-\beta)+\beta \alpha x] e^{-\alpha(1+\kappa) x},
$$

where

$$
w_{\tau, \kappa}=\gamma \alpha \frac{(1+\kappa) \bar{\gamma}^{\kappa}(\beta \alpha)^{\tau}}{(2-\beta)^{\tau+1}}
$$

\section{References}

1. Shaked, M.; Shanthikumar, J. Stochastic Orders; Springer: New York, NY, USA, 2007.

2. Barlow, R.E.; Proschan, F. Statistical Theory of Reliability and Life Testing; Holt, Rinehart, and Winston: New York, NY, USA, 1975.

3. Bakouch, S.; Jazi, M.; Nadarajah, S.; Dolati, A.; Roozegar, R. A lifetime model with increasing failure rate. Appl. Math. Model. 2014, 38, 5392-5406. [CrossRef] 
4. Marshall, A.W.; Olkin, I. A new method for adding a parameter to a family of distributions with application to the exponential and Weibull families. Biometrika 1997, 84, 641-652. [CrossRef]

5. Navarro, J.; Franco, M.; Ruiz, J.M. Characterization through moments of the residual life and conditional spacing. Indian J. Stat. Ser. A 1998, 60, 36-48.

6. Alizadeh, M.; Ghosh, I.; Yousof, H.M.; Rasekhi, M.; Hamedani, G.G. The generalized odd generalized exponential family of distributions: Properties, characterizations and applications. J. Data Sci. 2017, 15, 443-466.

7. Yousof, H.M.; Afify, A.Z.; Alizadeh, M.; Nadarajah, S.; Aryal, G.R.; Hamedani, G.G. The Marshall-Olkin generalized-G family of distributions with Applications. Statistica 2018, 78, 273-295.

8. Yousof, H.M.; Altun, E.; Hamedani, G.G. A new extension of Frechet distribution with regression models, residual analysis and characterizations. J. Data Sci. 2018, 16, 743-770.

9. Yousof, H.M.; Afify, A.Z.; Alizadeh, M.; Hamedani, G.G.; Jahanshahi, S.M.A.; Ghosh, I. The generalized transmuted Poisson-G family of Distributions. Pak. J. Stat. Oper. Res. 2018, 14, 759-779. [CrossRef]

10. Yousof, H.M.; Altun, E.; Ramires, T.G.; Alizadeh, M.; Rasekhi, M. A new family of distributions with properties, regression models and applications. J. Stat. Manag. Syst. 2018, 21, 163-188. [CrossRef]

11. Yousof, H.M.; Butt, N.S.; Alotaibi, R.; Rezk, H.; Alomani, G.A.; Ibrahim, M. A new compound Fréchet distribution for modeling breaking stress and strengths data. Pak. J. Stat. Oper. Res. 2019, 15, 1017-1035. [CrossRef]

12. Yousof, H.M.; Rasekhi, M.; Altun, E.; Alizadeh, M. The extended odd Frechet family of distributions: properties, applications and regression modeling. Int. J. Appl. Math. Stat. 2018, 30, 1-30.

13. Nascimento, A.D.C.; Silva, K.F.; Cordeiro, G.M.; Alizadeh, M.; Yousof, H.M. The odd Nadarajah-Haghighi family of distributions: Properties and applications. Stud. Scientiarum Math. Hungarica 2019, 56, 1-26. [CrossRef]

14. Bantan, R.A.R.; Jamal, F.; Chesneau, C.; Elgarhy, M. Truncated inverted Kumaraswamy generated family of distributions with applications. Entropy 2019, 21, 1089. [CrossRef]

15. Arshad, R.M.I.; Chesneau, C.; Jamal, F. The odd gamma Weibull-geometric model: Theory and applications. Mathematics 2019, 7, 399. [CrossRef]

16. Korkmaz, M.C.; Altun, E.; Yousof, H.M.; Hamedani, G.G. The odd power Lindley generator of probability distributions: Properties, characterizations and regression modeling. Int. J. Stat. Probab. 2019, 8, 70-89. [CrossRef]

17. Korkmaz, M.C.; Yousof, H.M.; Hamedani, G.G. The exponential Lindley odd log-logistic G family: properties, characterizations and applications. J. Stat. Theory Appl. 2018, 17, 554-571. [CrossRef]

18. Elsayed, H.A.H.; Yousof, H.M. The Burr X Nadarajah Haghighi distribution: Statistical properties and application to the exceedances of flood peaks data. J. Math. Stat. 2019, 15, 146-157. [CrossRef]

19. Merovci, F.; Alizadeh, M.; Yousof, H.M.; Hamedani, G.G. The exponentiated transmuted-G family of distributions: theory and applications. Commun. Stat.-Theory Methods 2017, 46, 10800-10822. [CrossRef]

20. Korkmaz, M.C.; Yousof, H.M.; Ali, M.M. Some theoretical and computational aspects of the odd Lindley Fréchet distribution. J. Stat.: Stat. Actuarial Sci. 2017, 2, 129-140.

21. Ibrahim, M.; Yadav, A.S.; Yousof, H.M.; Goual, H.; Hamedani, G.G. A new extension of Lindley distribution: modified validation test, characterizations and different methods of estimation. Commun. Stat. Appl. Methods 2019, 26, 473-495. [CrossRef]

22. Idika, E.; Anthony, C.; Johnson, O. Marshall-Olkin generalized Erlange-truncated exponential distribution: Properties and Applications. Cogent Math. 2017, 4, 1-19.

23. Hamedani, G.G.; Altun, E.; Korkmaz, M.C.; Yousof, H.M.; Butt, N.S. A new extended G family of continuous distributions with mathematical properties, characterizations and regression modeling. Pak. J. Stat. Oper. Res. 2018, 14, 737-758. [CrossRef]

24. Alizadeh, M.; Rasekhi, M.; Yousof, H.M.; Hamedani, G.G. The transmuted Weibull G family of distributions. Hacet. J. Math. Stat. 2018, 47, 1-20. [CrossRef]

25. Yadav, A.S.; Goual, H.; Alotaibi, R.M.; Rezk, H.; Ali, M.M.; Yousof, H.M. Validation of the Topp-Leone-Lomax model via a modified Nikulin-Rao-Robson goodness-of-fit test with different methods of estimation. Symmetry 2020, 12, 57. [CrossRef]

26. Hamedani, G.G.; Rasekhi, M.; Najibi, S.M.; Yousof, H.M.; Alizadeh, M. Type II general exponential class of distributions. Pak. J. Stat. Oper. Res. 2019, 15, 503-523. [CrossRef] 
27. Hamedani, G.G.; Yousof, H.M.; Rasekhi, M.; Alizadeh, M.; Najibi, S.M. Type I general exponential class of distributions. Pak. J. Stat. Oper. Res. 2017, 14,39-55. [CrossRef]

28. Ibrahim, M. A new extended Fréchet distribution: Properties and estimation. Pak. J. Stat. Oper. Res. 2019, 15, 773-796.

29. Alizadeh, M.; Yousof, H.M.; Rasekhi, M.; Altun, E. The odd log-logistic Poisson-G Family of distributions. J. Math. Ext. 2019, 12, 81-104.

30. Ghitany, M.E.; Al-Awadhi, F.A.; Alkhalfan, L.A. Marshall-Olkin extended Lomax distribution and its application to censored data. Commun. Statist. Theor. Meth. 2007, 36, 1855-1866. [CrossRef]

31. Aryal, G.R.; Yousof, H.M. The exponentiated generalized-G Poisson family of distributions. Econ. Qual. Control 2017, 32, 1-17. [CrossRef]

(C) 2020 by the authors. Licensee MDPI, Basel, Switzerland. This article is an open access article distributed under the terms and conditions of the Creative Commons Attribution (CC BY) license (http://creativecommons.org/licenses/by/4.0/). 